\title{
IPOL: a new journal for fully reproducible research; analysis of four years development
}

\section{Colom ${ }^{1}$, B. Kerautret ${ }^{2}$, N. Limare ${ }^{1}$, P. Monasse ${ }^{3}$, and J.M. Morel ${ }^{1}$}

${ }^{1}$ CMLA, ENS Cachan ${ }^{2}$ LORIA, Univ. de Lorraine ${ }^{3}$ LIGM, Univ. Paris-Est
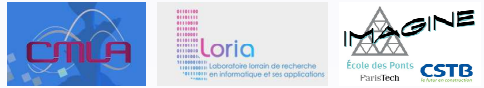

Worskshop RCBR 2015, NTMS 2015 July 29, Paris

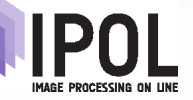




\section{The IPOL Journal: context of reproductible research (1)}

Reproductible research in sciences:

- Theoretical scientists share demonstrations; 


\section{The IPOL Journal: context of reproductible research (1)}

Reproductible research in sciences:

- Theoretical scientists share demonstrations;

- Experimental scientist share procedures; 


\section{The IPOL Journal: context of reproductible research (1)}

Reproductible research in sciences:

- Theoretical scientists share demonstrations;

- Experimental scientist share procedures;

- Computational scientists... ? 


\section{The IPOL Journal: context of reproductible research (1)}

Reproductible research in sciences:

- Theoretical scientists share demonstrations;

- Experimental scientist share procedures;

- Computational scientists... ?

Computer Science:

- Description of methods/algorithms;

- description often limited (constraints on page limits);

- parameters not given or not well described;

- steps of pre/post processing missing. 


\section{The IPOL Journal: context of reproductible research (2)}

Research in Computer Science:

1 New idea;

2 demonstration, implementation;

3 article publication.

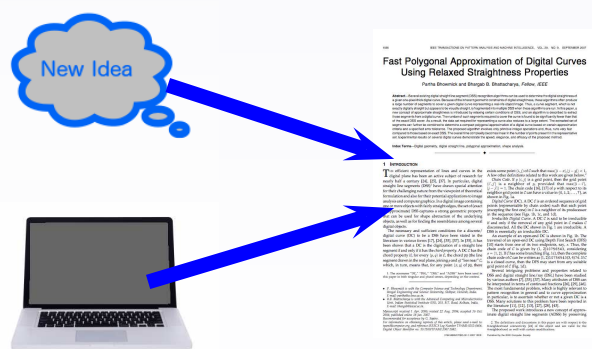




\section{The IPOL Journal: context of reproductible research (2)}

Research in Computer Science:

1 New idea;

2 demonstration, implementation;

3 article publication.
Reusable Research:

1 Article which seems interesting;

2 re-implement the algorithm;

3 conformity of the results with the original.
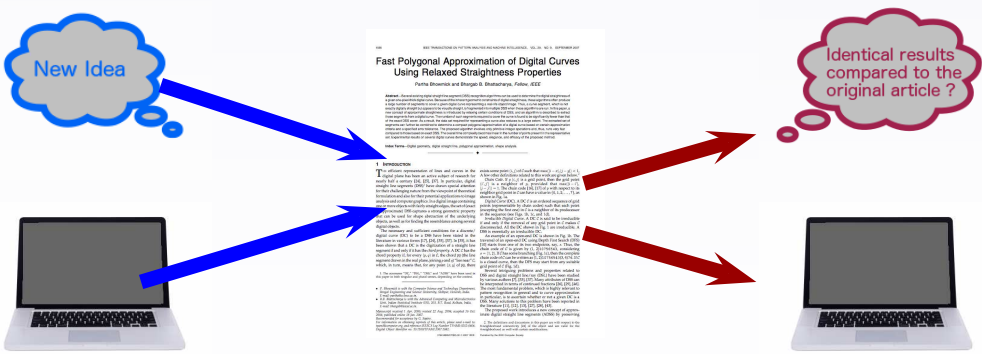


\section{The IPOL Journal: context of reproductible research (3)}

Frequent difficulties in computer science (image processing):

- Source code often not available (or not reviewed);

- quality/stability of the results not easy to analyze;

- testing with different input data not possible. 


\section{The IPOL Journal: context of reproductible research (3)}

Frequent difficulties in computer science (image processing):

- Source code often not available (or not reviewed);

- quality/stability of the results not easy to analyze;

- testing with different input data not possible.

Consequences:

- Comparisons and experiments difficult;

- potential time loss for the reader;

- limits the diffusion of research. 


\section{The IPOL Journal: context of reproductible research (4)}

Providing source code/data

- $\oplus$ A real added value for the publication;

- $\oplus$ increases the impact/comparisons;

- $\ominus$ software is not really acknowledged;

- $\ominus$ important effort (documentation, tests, user maintenance). 


\section{The IPOL Journal: context of reproductible research (4)}

Providing source code/data

- $\oplus$ A real added value for the publication;

- $\oplus$ increases the impact/comparisons;

- $\ominus$ software is not really acknowledged;

- $\ominus$ important effort (documentation, tests, user maintenance).

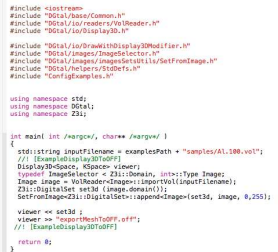

Software Diffusion

- Specialized journals in software:

Source Code for Biology and Medicine, Journal of Open Research Software, Computing in Science and Engineering, ...

- Diffusion platform:

RunMyCode / Run\&Share, Figshare, DataDryad, Harvard Dataverse, Ubiquity Metajournals, Zenodo (with doi) ...

$\Rightarrow$ no validation, no scientific review (reliability and durability problem). 


\section{The IPOL Journal: origin and motivation}

Origin:

- Journal started in October 2009;

- under the initiative of Nicolas Limare, Jean-Michel Morel and the Image Processing team at the CMLA lab (ENS-Cachan);

- first article published in 2010. 


\section{The IPOL Journal: origin and motivation}

Origin:

- Journal started in October 2009;

- under the initiative of Nicolas Limare, Jean-Michel Morel and the Image Processing team at the CMLA lab (ENS-Cachan);

- first article published in 2010.

Motivation [Limare \& Morel 2009]:

- Reproductible research;

- new way to publish research results;

- allows everybody to test the algorithms;

$\Rightarrow$ with their own images

- independent of the platform (the demos execute on the server side and the results are shown to the user using a web interface). 


\section{IPOL Journal: principle and current form (1)}

Characteristics:

- Research journal in image processing;

- each article contains a description of one algorithm and its source code;

- association of each article with its online demonstration, with archived experiments;

- the peer-review process includes the article, demo, and source code;

- Open Science journal and Reproductible Research.

\section{IPOL Journal · Image Processing On Line}

HOME - ABOUT - ARTICLES - PREPRINTS - WORKSHOPS - NEWS - SEARCH

IPOL is a research journal of image processing and image analysis which emphasizes the role of mathematics as a source for algorithm design and the reproducibility of the research. Each article contains a text on an algorithm and its source code, with an online demonstration facility and an archive of experiments. Text and source code are peer-reviewed and the demonstration is controlled. IPOL is an Open Science and Reproducible Research journal.

A Editorial Policy \& Editorial Board Submit an Article $\square$ Follow IPOL

Index · Articles 20112012201320142015 · Preprints · News · Citation score 


\section{IPOL Journal: principle and current form (2)}

Philisophy of the journal:

- Follows the guideline on reproductible research topics;

- reproductible research standard [Stodden 09a] [Stodden 09b];

- answer to credibility crisis in scientific computation (as pointed out by Donoho [Donoho et al. 09]). 


\section{IPOL Journal: principle and current form (2)}

Philisophy of the journal:

- Follows the guideline on reproductible research topics;

- reproductible research standard [Stodden 09a] [Stodden 09b];

- answer to credibility crisis in scientific computation (as pointed out by Donoho [Donoho et al. 09]).

What IPOL is not:

- IPOL publishes algorithms along with their implementation, but not compiled software;

- IPOL is not a software library (each code has minimal dependencies);

- IPOL is not a software or code diffusion platform. 


\section{IPOL Journal: principle and current form (3)}

- Current form: "classic" (with online PDF).

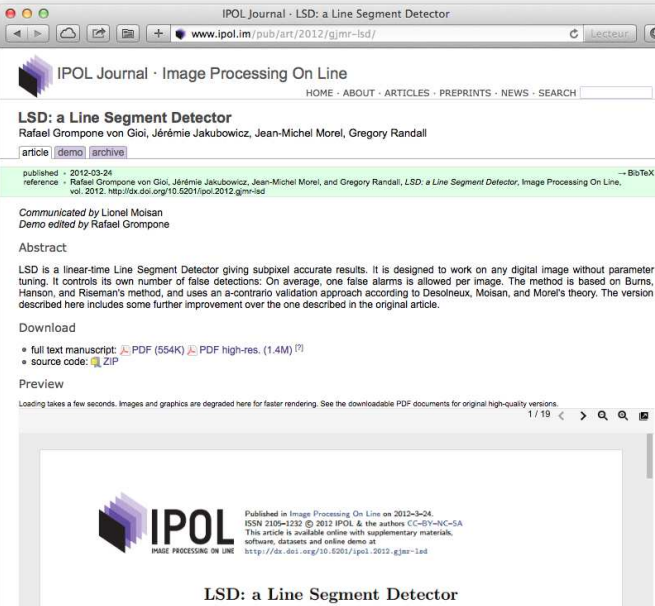

LSD: a Line Segment Detector 


\section{IPOL Journal: principle and current form (3)}

- Current form: "classic" (with online PDF).

- Associated demos.

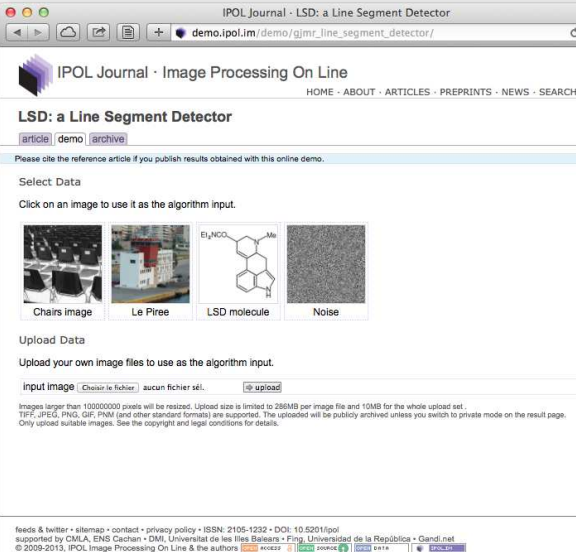

Chairs image

Upload Data

Upload your own irnage fles to use as the agorithm input.

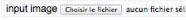

Supioat

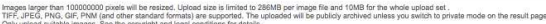

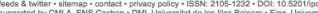

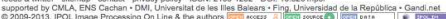




\section{IPOL Journal: principle and current form (3)}

- Current form: "classic" (with online PDF).

- Associated demos.

- Archive containing experiments with data uploaded by users.

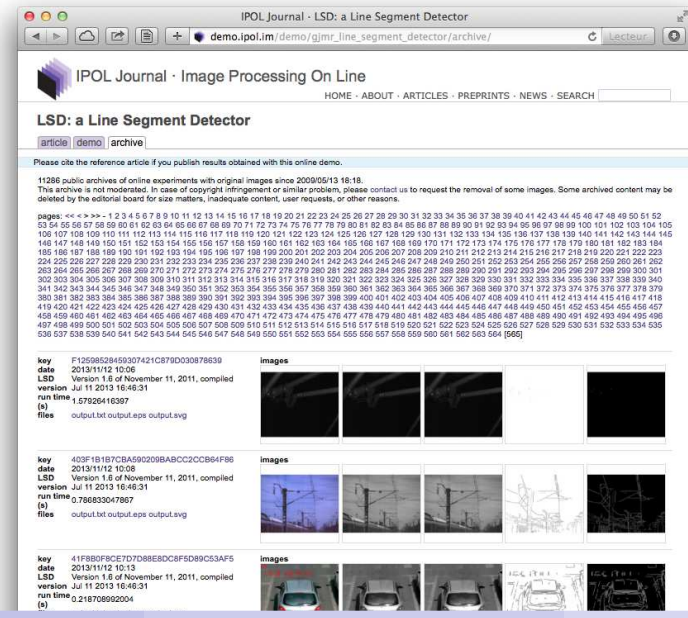




\section{IPOL Journal: editorial structure}

Same aspects as a classical journal:

- Editorial project, editorial committee;

- articles, authors, editors;

- reviewing process and validation;

- ISSN, DOI;

- special issues;

- currently indexed by:

Scirus, Google Scholar, DBLP, DOAJ, SHERPA/ROMEO, Héloïse, WorldCat, CrossRef, Ulrich, Index Copernicus, PBN, JGate, VisionBib, CVonline, JournalSeek, and NewJour. 


\section{IPOL Journal: editorial structure}

Same aspects as a classical journal:

- Editorial project, editorial committee;

- articles, authors, editors;

- reviewing process and validation;

- ISSN, DOI;

- special issues;

- currently indexed by:

Scirus, Google Scholar, DBLP, DOAJ, SHERPA/ROMEO, Héloïse, WorldCat, CrossRef, Ulrich, Index Copernicus, PBN, JGate, VisionBib, CVonline, JournalSeek, and NewJour.

Software point of view:

- Each article should propose an implementation;

- reviewing step, verification, validation, and publication;

- reviewer: check the correspondence between the algorithm description in the article and code (+ code readability and code documentation). 
2. Scientific \& technical achievements to establish a state of the art (1)

Image Denoising

- Papers on image denoising cover most of the state of the art in image denoising. $\Rightarrow$ analyze and finalize the often incomplete algorithms.

- Online implementation allows the first objective comparison.

- complete state of the art of denoising

$\Rightarrow$ See the noise Clinic [Lebrun et al. 15] 


\section{Scientific \& technical achievements to establish a state of the art (1)}

\section{Image Denoising}

- Papers on image denoising cover most of the state of the art in image denoising. $\Rightarrow$ analyze and finalize the often incomplete algorithms.

- Online implementation allows the first objective comparison.

- complete state of the art of denoising

$\Rightarrow$ See the noise Clinic [Lebrun et al. 15]

Stereovision

The stereovision category at IPOL contains five fundamental algorithms:

- Quasi-Euclidean Epipolar Rectification [Monasse 11].

- Kolmogorov \& Zabih's graph cuts stereo matching algorithm [Kolmogorov et al. 14].

- Stereo disparity through cost aggregation with guided filter [Tan \& Monasse 14].

- Integral images for block matching [Facciolo et al. 14].

- Bilaterally weighted patches for disparity map computation [Fernandez \& Monasse 15].

$\Rightarrow$ Others in preparation. 


\section{Scientific \& technical achievements to establish a state of the art (2)}

\section{Stereovision}

- First workshop demo delivering a 3D digital elevation model of the ground from satellite images.

- available here: http://dev.ipol.im/ carlo/ipol_demo/workshop_s2p 


\section{Technical Issues Overcome Through the Development of IPOL}

First problem: reference programming languages

The chosen languages must:

- have a stable API;

- be used intensively by reasearchers and the industry;

- have stable standard libraries. 


\section{Technical Issues Overcome Through the Development of IPOL}

First problem: reference programming languages

The chosen languages must:

- have a stable API;

- be used intensively by reasearchers and the industry;

- have stable standard libraries.

Accepted languages and libraries

- First accepted languages: ANSI C/C++;

- currently accepted libraries: libtiff, libjpeg, libpng, zlib, FFTW, GSL, Eigen, cblas, and clapack;

- currently accepted MATLAB toolboxes: Image Processing, Optimization, Wavelet;

- new accepted frameworks: MATLAB, Python (with NumPy and SciPy);

- other languages might be considered and accepted. 
Second problem: design an online demo system

A demo system was created for this purpose, from scratch. It has to manage the execution of each online experiment:

- The parameters and result pages are different in each demo;

- the test images are different in each demo;

- users must be able to upload their own images;

- it has to be fast enough to allow online execution $\rightarrow$ Multiple CPU system;

- it has to archive user experiments (input data, input parameters, and results). 


\section{Second problem: design an online demo system}

A demo system was created for this purpose, from scratch. It has to manage the execution of each online experiment:

- The parameters and result pages are different in each demo;

- the test images are different in each demo;

- users must be able to upload their own images;

- it has to be fast enough to allow online execution $\rightarrow$ Multiple CPU system;

- it has to archive user experiments (input data, input parameters, and results).

\section{Problems detected in the current system}

The current system is usable and functional, but we detected several problems:

- Creating a new demo implies coding in Python and designing HTML templates;

- non-scalable, too complex internal structure, and with lack of modularization.

$\Rightarrow$ Now moving towards a modular system with automatic demo generation. 


\section{Problems in the current demo system}

- Most of the problems related to the architecture of the system;

- system designed as an object-oriented monolithic kernel;

- too complex, with tightly interface-coupled components;

- non-scalable;

- not easy to distribute the system over different machines. 


\section{Problems in the current demo system}

- Most of the problems related to the architecture of the system;

- system designed as an object-oriented monolithic kernel;

- too complex, with tightly interface-coupled components;

- non-scalable;

- not easy to distribute the system over different machines.

Improvements

We're currently working on improving the system

- Modular architecture:

- Specialized standalone modules;

- the monolithic kernel becomes a simple controller;

- the core controller and the modules communicate via webservices.

- Automatic demo generation:

- Each demo is specified using a simple textual description (name, type, and default value of the parameters and format of result page);

- no need to code or design any page to build a new demo. 
Quality of articles and a "natural selection"

Most failed IPOL projects aborted when:

- the described algorithm was incomplete;

- did not give all the results described in the paper;

- run time not reasonable;

- worked only on a certain type of data. 
Progress towards the establishment of a full state of the art in each of the main sections of the journal

Identify algorithms representing a very substantial portion of the state of the art:

- Most efficient algorithms should be published;

- proposed theories and methods, even though they are not (or no longer) considered the best. 
Progress towards the establishment of a full state of the art in each of the main sections of the journal

Identify algorithms representing a very substantial portion of the state of the art:

- Most efficient algorithms should be published;

- proposed theories and methods, even though they are not (or no longer) considered the best.

Some sections currently in IPOL

- Color and Contrast (10 articles)

- Denoising (15 articles)

- Demosaicking (6 articles)

- Interpolation (4 articles)

- Optical Flow (6 articles)

- Vision Through Turbulence (3 articles) 
Goal: cover must of their respective state of the art (performance and descriptions of main mathematical techniques)

Others sections

- 3D

- Blur

- Computational Photography

- Geometry

- Infrared

- Learning and Detection

- Inpainting

- Image Comparison

- PDE

- Stereovision

- Texture

$\rightarrow$ However, still incomplete! 
Example of advantages of a reproducible algorithms with Stereovision: Middlebury stereo evaluation

- One method [Fernandez \& Monasse 15] was implemented from an algorithm on adaptive neighborhoods [?];

- the obtained results were different!

- it was discovered that a post-processing must be applied to the main algorithm;

- there is no mention of a post-processing in the original article [?]! 


\section{Criticism to IPOL}

- Excessive effort (rigor, run on any data, code description);

- excessive length of the peer review report (including code review: slow and demanding);

- large number of objects to be published (article, source code, demo);

- no official impact factor;

- frustrating to work on algorithms designed by others;

- restricted number of authorized libraries or toolboxes (as libjpeg, libtiff, FFTW, MATLAB Image Processing toolbox,...) 
Authors and publishers praised IPOL for:

- Immediate impact of their publication;

- impact due to the very existence of the demo;

- gain tangible industrial and academic credibility;

- facilitate obtaining research funding (ERC, ONR, ANR, DGA, CNES, FUI, ...) 
Conclusions ( $($ )

- Reproducible Research redefines the output of the research: not only the article, but also the source code and the data.

- IPOL is a complete and fully functional Reproducible Research journal: articles, demos, data. Everything is free or open source.

- For the authors it takes more effort to write Reproducible Research articles, but: $\Rightarrow$ benefit is immediate (credibility and number of citations);

$\Rightarrow$ for both the IPOL article and the one published in a different journal with a demo available in IPOL).

- It is important to adapt the journal to the community needs and usages:

$\Rightarrow$ accept commonly used languages, libraries, and frameworks. 


\section{Conclusions (II)}

Pseudo-code is the main production of IPOL over the source code itself:

- The pseudo-code describes the significant parts of the algorithm;

- it does not contain all the details needed to encode it using an actual programming language;

- the pseudo-code is aimed to be readable, and reusable;

$\Rightarrow$ in general, it is between one and two orders of magnitude shorter than the actual source code.

- the pseudo-code is unambiguous to the mathematician reader. 


\section{What is Next? (I)}

\section{Facts:}

- More than 5000 articles/year on Computer Vision and Image processing describing algorithms;

- however, about 200 of these articles would we enough to cover the CV and IP state of the art. Less than 400 if exhaustive.

IPOL is producing 40 articles/year $\rightarrow$ It can exhaust the state of the art (old and new) in about 7 years. 


\section{What is Next? (II)}

Moving towards a new way to do research

- What is next, then? $\rightarrow$ Focus on incremental research.

- Antecedents: analysis of the genome.

- Cycle:

(1) review estabilished and published algoritms;

(8) combine them;

(3) improve them to achieve new and better results and applications.

In definitive, a new methodology to do research in CV and IP 


\section{Acknowledgement}

Work partly founded by the European Research Council (advanced grant Twelve Labours), the Office of Naval Research (ONR grant N00014-14-1-0023), and ANR-DGA project ANR-12-ASTR-0035. 


\section{References I}

[Stodden 09a] V. Stodden

The legal framework for reproducible scientific research: Licensing and copyright

Computing in Science \& Engineering, vol. 11, no. 1, pp. 35-40, 2009.

[Stodden 09b] V. Stodden

Enabling reproducible research: Open licensing for scientific innovation

International Journal of Communications Law and Policy, Forthcoming, 2009.

高

[Buckheit \& Donoho 95] J. B. Buckheit and D. L. Donoho

Wavelab and reproducible research

Springer, 1995.

[Gentleman 05] R. Gentleman

Reproducible research: A bioinformatics case study

Statistical applications in genetics and molecular biology, vol. 4, no. 1, 2005.

言

[Donoho et al. 09] D. L. Donoho, A. Maleki, I. U. Rahman, M. Shahram, and V. Stodden

Reproducible research in computational harmonic analysis

Computing in Science \& Engineering, vol. 11, no. 1, pp. 8-18, 2009.

[Donoho 10] D. L. Donoho

An invitation to reproducible computational research

Biostatistics, vol. 11, no. 3, pp. 385-388, 2010.

[Lebrun et al. 15] M. Lebrun, M. Colom, and J.-M. Morel

The Noise Clinic: a Blind Image Denoising Algorithm,

Image Processing On Line, vol. 5, pp. 1-54, 2015. 


\section{References II}

[Monasse 11] P. Monasse

Quasi-Euclidean Epipolar Rectification

Image Processing On Line, vol. 1, 2011.

[Kolmogorov et al. 14] V. Kolmogorov, P.Monasse and P.Tan

Kolmogorov and Zabih's Graph Cuts Stereo Matching Algorithm

Image Processing On Line, vol. 4, pp. 220-251, 2014.

[Tan \& Monasse 14] P. Tan and P. Monasse

Stereo Disparity through Cost Aggregation with Guided Filter

Image Processing On Line, vol. 4, pp. 252-275, 2014.

[Facciolo et al. 14] G. Facciolo, N. Limare, and E. Meinhardt-Llopis

Integral Images for Block Matching, Image Processing On Line, vol. 4, pp. 344-369, 2014.

[Fernandez \& Monasse 15] L. Fernández Julià and P. Monasse

Bilaterally Weighted Patches for Disparity Map Computation

Image Process- ing On Line, vol. 5, pp. 73-89, 2015.

[Limare \& Morel 11] N. Limare and J.-M. Morel

The ipol initiative: Publishing and testing algorithms on line for reproducible research in image processing Procedia Computer Science, vol. 4, pp. 716-725, 2011.

[Gamma et al. 94] E. Gamma, R. Helm, R. Johnson and J. Vlissides

Design patterns: elements of reusable object-oriented software

Pearson Education, 1994. 


\section{References III}

[Yoon \& Kweon 06] K.-J. Yoon and I. Kweon Adaptive support-weight approach for correspondence search IEEE Transactions on Pattern Analysis and Machine Intelligence, vol. 28, no. 4, pp. 650-656, 2006.

量

[CGAL] Computational Geometry Algorithms Library,

http://www.cgal.org

[DGtal] DGtal: Digital Geometry tools and algorithms library, http://libdgtal.org

[Limare \& Morel 2009] Limare N. and Morel, J-M (2009) IPOL Project Presented at the CMLA Seminar http://www.ipol.im/news/20091022_cmla/s5.html CMLA ENS Cachan 


\section{IPOL citations (07/28/2015)}

\section{IPOL CITATIONS}

IPOL Journal Image Processing online

Image Processing, Applied Mathematics

Verified email at ctim.es - Homepage

\begin{tabular}{|c|c|c|}
\hline Title $1-20$ & Cited by & Year \\
\hline 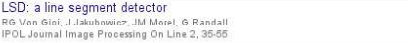 & $460 *$ & 2012 \\
\hline $\begin{array}{l}\text { Asif: An algorithm for fully affine invariant comparison } \\
\text { o Yui JM Morel } \\
\text { IPoL Journal Image Frocessing on Line } 1\end{array}$ & 80 & 2011 \\
\hline $\begin{array}{l}\text { Non-Local Means Denoising } \\
\text { A Buades, B Coll, JM Norel } \\
\text { IPOL Journal Image Processing on Line } 1\end{array}$ & 52 & 2011 \\
\hline $\begin{array}{l}\text { Rudin-Osher-Fatemi total variation denoising using split Bregman } \\
\text { P Getreuet } \\
\text { IPOL Joumal : Image Processing On Line 2, 79-95 }\end{array}$ & 48 & 2012 \\
\hline $\begin{array}{l}\text { An Analysis and Implementation of the BM3D Image Denoising Method } \\
\text { M Lebrun } \\
\text { IPOL Joumal Image Processing On Line } 2 ; 175-213\end{array}$ & 32 & 2012 \\
\hline $\begin{array}{l}\text { DCT image denoising: a simple and effective image denoising algorithm } \\
\text { o Yu, Q Sapiro } \\
\text { IPOL Journal Image Proocsing on Line } 1\end{array}$ & 30 & 2011 \\
\hline $\begin{array}{l}\text { Implementation of the "Non-Local Bayes" (NL-Bayes) Image Denoising } \\
\text { Algorithm } \\
\text { M Lebrun, A Buades, Jil Morel } \\
\text { IPOL Journal Image Processing on Line } 3,1-42\end{array}$ & 25 * & 2013 \\
\hline $\begin{array}{l}\text { TV-L1 Optical Flow Estimation } \\
\text { J Sanchez, E Meinharct-Llopis, G Faociolo } \\
\text { IPOL Joumal Image Processing On Line. 3: 137-150 }\end{array}$ & 24 * & 2013 \\
\hline $\begin{array}{l}\text { Horn-schunck optical flow with a multi-scale strategy } \\
\text { E Meinhardt-Llopis, JS Perez, D Kondermann } \\
\text { IPOL Journal-Imags Proosssing on Line } 3,151,172\end{array}$ & 19 & 2013 \\
\hline $\begin{array}{l}\text { Automatic homographic registration of a pair of images, with a contrario } \\
\text { elimination of outliers } \\
\text { LMoisan, P Moulon, P Monasse } \\
\text { IPOL Journal I Image Processing on Line } 2,56-73\end{array}$ & 19 & 2012 \\
\hline
\end{tabular}

\section{Google Scholar}

Get my own profile

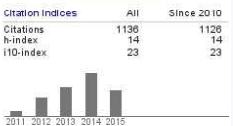




\section{IPOL citations (07/28/2015)}

\begin{tabular}{|c|c|c|}
\hline $\begin{array}{l}\text { E Meinhardt-Llopis, JS Pèrez, D Kondermann } \\
\text { IPOL Journal Image Processing On Line } 3,151-172\end{array}$ & 19 & 2013 \\
\hline $\begin{array}{l}\text { Automatic homographic registration of a pair of images, with a contrario } \\
\text { elimination of outliers } \\
\text { LMoisan, P Mouton, P Monasse } \\
\text { IPOL Joumal Image Processing On Line 2, 58-73 }\end{array}$ & 19 & 2012 \\
\hline $\begin{array}{l}\text { An analysis of the SURF method } \\
\text { E Oyallon, J Rabin } \\
\text { IPOL Journal Image Processing On Line } 5(2015), 170-218\end{array}$ & $18 *$ & 2015 \\
\hline $\begin{array}{l}\text { An Implementation and Detailed Analysis of the K-SVD Image Denoising } \\
\text { Algorithm } \\
\text { M Letum, A Letatire } \\
\text { IPOL Journal Image Processing Online 2, } 86-133\end{array}$ & 18 * & 2012 \\
\hline $\begin{array}{l}\text { Total variation deconvolution using split Bregman } \\
\text { P Getreuer } \\
\text { IPOL Journal Image Processing on Line } 2 \text { (1), 158-174 }\end{array}$ & 17 & 2012 \\
\hline $\begin{array}{l}\text { Automatic color enhancement (ACE) and its fast implementation } \\
\text { P Getreuer } \\
\text { IPOL Journal : Image Processing On Line 2, 288-277 }\end{array}$ & 14 & 2012 \\
\hline $\begin{array}{l}\text { Algebraic Lens Distortion Model Estimation } \\
\text { LAlvarez, LGomez, JR Sendra } \\
\text { IPot Lournal Image Procassing On I ine } 1\end{array}$ & 13 & 2011 \\
\hline $\begin{array}{l}\text { Chan-vese segmentation } \\
\text { P Getreuer } \\
\text { IPOL Journal Image Processing on Line 2, } 214224\end{array}$ & 12 & 2012 \\
\hline $\begin{array}{l}\text { Simplest color balance } \\
\text { N Limare, JL Lisani, JM Morel, AB Petro, o Sbert } \\
\text { HroL Journal Image Hrocessing un Line } 1\end{array}$ & 12 & 2011 \\
\hline $\begin{array}{l}\text { An Analysis of the Viola-Jones Face Detection Algorithm } \\
\text { YQ Wang } \\
\text { IPOL Joumal Image Processing On Line 4, 128-148 }\end{array}$ & 11 & 2014 \\
\hline $\begin{array}{l}\text { Exemplar-based Texture Synthesis: the Efros-Leung Algorithm } \\
\text { o Aguerrebers. Y Gousseau, \& Tartawel } \\
\text { IPOL Journal Image Processing On Line 3, 213-231 }\end{array}$ & 11 & 2013 \\
\hline $\begin{array}{l}\text { Micro-texture synthesis by phase randomization } \\
\text { B Galeme, Y Gousseau, JM Moref } \\
\text { IPOL Journal Image Processing On Line } 1\end{array}$ & 11 & 2011 \\
\hline
\end{tabular}

